\title{
Analytical modeling of the gas-filling dynamics in photonic crystal fibers
}

\author{
Isabelle Dicaire, ${ }^{*}$ Jean-Charles Beugnot, and Luc Thévenaz \\ École Polytechnique Fédérale de Lausanne (EPFL), Institute of Electrical Engineering, \\ STI-GR-SCI-LT Station 11, 1015 Lausanne, Switzerland \\ ${ }^{*}$ Corresponding author: isabelle.dicaire@epfl.ch
}

Received 14 May 2010; accepted 12 July 2010; posted 23 July 2010 (Doc. ID 128481); published 17 August 2010

\begin{abstract}
We present useful expressions predicting the filling time of gaseous species inside photonic crystal fibers. Based on the theory of diffusion, this gas-filling model can be applied to any given fiber geometry or length by calculating diffusion coefficients. This was experimentally validated by monitoring the filling process of acetylene gas in several fiber samples of various geometries and lengths. The measured filling times agree well, within $\pm 15 \%$, with the predicted values for all fiber samples. In addition, the pressure dependence of the diffusion coefficient was experimentally verified by filling a given fiber sample with acetylene gas at various pressures. Finally, optimized conditions for gas-light interaction are determined by considering the gas flow dynamics in the design of microstructured fibers for gas detection and all-fiber gas cell applications. (C) 2010 Optical Society of America
\end{abstract}

OCIS codes: $\quad 060.5295,290.1990$.

\section{Introduction}

Photonic crystal fibers (PCFs) are now widely used optical sensing tools as they offer the possibility to make interactions between light guided in the fiber and molecules trapped inside the capillaries. In addition to the high interaction length that is obtained in such small sensing volumes, PCFs can be coiled up into very small diameters due to their high robustness. Combined with the ability to engineer their physical and optical properties, PCFs are attractive candidates for realizing chemical sensors and gas cells [1-ㅣ] . For instance, solid-core microstructured fibers can be designed to have specific dispersion characteristics or to have maximum overlap between the optical field and the gas species [4,5]. Applications such as acetylene gas spectroscopy and liquid chemical sensing have been successfully performed in suspended-core fibers where up to $29 \%$ of the optical power is available for sensing via the evanescent field [6,7]. On the other hand, hollow-core fibers are usually preferred for weak absorption lines

0003-6935/10/244604-06\$15.00/0

(C) 2010 Optical Society of America or short fiber lengths, even considering their limited bandwidths and low design flexibility, since the overlap between the optical field and the sensing species is nearly complete [8].

In addition to the fraction of optical power available for sensing, the filling time is also an important parameter to consider when designing a fiber-based gas sensor since the microscopic size of the holes may lead by capillarity to an endless gas progression along the fiber. Previous papers monitored the gasfilling process in hollow-core fibers using acetylene absorption lines and studied its dependence on fiber length [9-11]. Accurate expressions have been provided to predict the temporal response of a gas sensor, but proved to be quite inconvenient due to the nonanalytical nature of the solutions [12]. This work aims at providing simple equations that can be applied to any type of fiber, fiber geometry, or length. Our model for the filling time is based on the theory of gas diffusion where approximations have been made to mitigate the complexity of the equations. The validity of these approximations is verified by analyzing the gas-filling dynamics in fiber samples of various geometries. Using this gas-filling model, 
the filling time can be predicted for any given fiber geometry and length.

\section{Theory of Gas Diffusion}

This section presents the equations governing the diffusion of gas molecules inside capillaries. We define the diffusive flux $\Phi$ as the amount of gas passing perpendicularly through a reference surface of unit area per unit time. It is directly a function of the gradient of the molecule density $n$ at a position $x$ :

$$
\Phi=-D \frac{\partial n}{\partial x}
$$

where the proportionality constant $D$ is the so-called diffusion coefficient. In addition, the conservation of mass during diffusion yields

$$
\frac{\partial n}{\partial t}=-\frac{\partial \Phi}{\partial x} .
$$

The diffusive flux $\Phi$ and the diffusion coefficient $D$ can be calculated; however, because the nature of the flow is completely different according to the experimental conditions, the corresponding diffusion regime must be identified (see Fig. 1). This is done by the means of a dimensionless parameter called the Knudsen number $K_{n}$, which is the mean free path $\lambda$ of a molecule divided by the capillary radius $a$ through which it is diffusing:

$$
K_{n}=\lambda / a, \quad \lambda=\frac{k_{B} T}{\sqrt{2} \pi \bar{P} \delta^{2}},
$$

where $k_{B}$ is the Boltzmann constant, $T$ is the temperature, $P$ is the average pressure along the fiber, and where the added bar sign indicates averaging over the filling time, as well. Finally, $\delta$ is the diam- eter of the gas molecule (334 pm for the acetylene molecule as defined in [12]).

\section{A. Free Molecular Flow Regime}

The low-pressure range of gas experiments is represented by large Knudsen numbers $\left(K_{n}>1\right)$ and the corresponding diffusion regime is called free molecular flow or, simply, molecular flow. In this regime, the gas molecules collide more frequently with the capillary walls than with other molecules. The molecular flux averaged over the cross section of the capillary is therefore determined by geometrical considerations:

$$
\Phi_{k}=-\frac{2}{3} \frac{a \bar{v}}{k_{B} T} \frac{\partial p}{\partial x},
$$

where the mean molecular velocity $\bar{v}$ is a function of both the temperature and the molecular mass $m$ :

$$
\bar{v}=\sqrt{\frac{8 k_{B} T}{\pi m}} .
$$

It is then straightforward to derive the diffusion coefficient for the free molecular flow regime $D_{k}$ from its definition in Eq. (1):

$$
D_{k}=\frac{2}{3} a \bar{v} .
$$

\section{B. Hydrodynamic Flow Regime}

On the other hand, small Knudsen numbers $\left(K_{n}<0.01\right)$ indicate that collisions between molecules occur more frequently than with the walls, which is the case inside a PCF at atmospheric pressure down to a hole size of $16 \mu \mathrm{m}$. In this situation, the flow velocity is parabolic across the fiber hole and tends to be zero at the fiber walls due to frictional forces. The gas flow is then considered viscous and

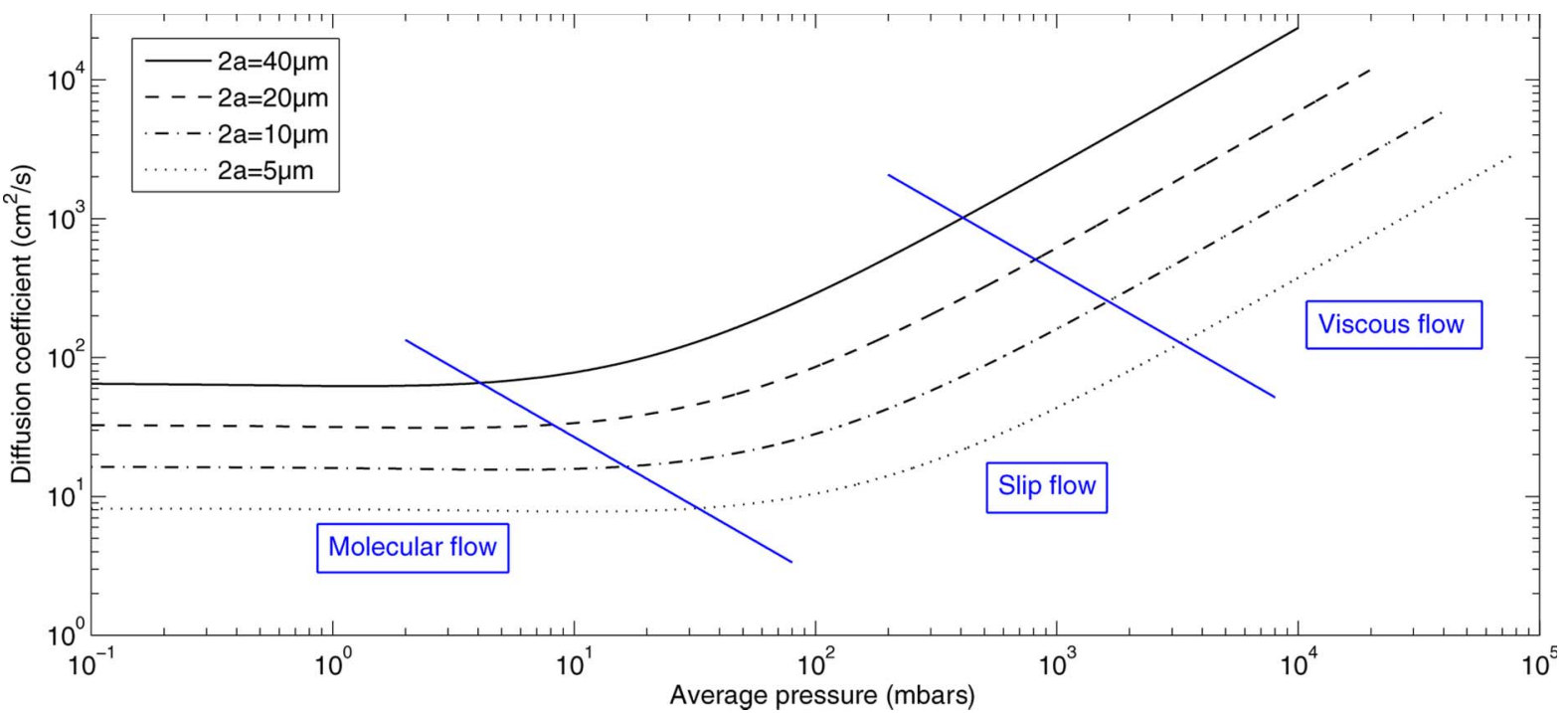

Fig. 1. (Color online) Calculated diffusion coefficients of acetylene gas for several capillary diameters. The various flow regimes are defined according to the two blue diagonal lines representing Knudsen numbers of 1 and 0.01 . 
is governed by hydrodynamical equations. More specifically, the Poiseuille equation describes a laminar and incompressible flow in the viscous range,

$$
\Phi_{v}=-\frac{a^{2} n}{8 \eta} \frac{\partial p}{\partial x}
$$

with $\eta$ being the viscosity,

$$
\eta=\frac{\bar{v} m}{2 \sqrt{2} \pi \delta^{2}} .
$$

The diffusion coefficient for the viscous flow regime is then obtained using Eq. (1):

$$
D_{v}=\frac{a^{2} p}{8 \eta} .
$$

\section{Slip-Flow Regime}

Between these two flow regimes is found a transition type of flow called slip flow that shows characteristics of both viscous and molecular flows. As illustrated in Fig. 1, the extrapolation of the viscous flow curves into the lower part of the transition region underestimates the actual flow. The additional flow can be seen as gas slipping over the capillary walls: the flow velocity at the walls is no longer zero, as in the viscous regime. There are no analytical expressions describing the slip-flow transition region so we had to rely on semiempirical relations.

The behavior of the diffusive flux $\Phi$ as a function of pressure can be described by using an empirical relation, $Z\left(K_{n}\right)$, scaling the flow additional to the extrapolated viscous flow $\Phi_{v}[\underline{13}, \underline{14}]$ :

$$
\Phi=\Phi_{v}+Z\left(K_{n}\right) \Phi_{k}
$$

The corresponding diffusion coefficient is

$$
D=\frac{a^{2} p}{8 \eta}+Z\left(K_{n}\right) \frac{2}{3} a \bar{v}
$$

where $Z\left(K_{n}\right)$ is

$$
Z=\frac{1+2.507(a / \lambda)}{1+3.095(a / \lambda)}=\frac{1+2.507 / K_{n}}{1+3.095 / K_{n}} .
$$

This way, at low pressures, $Z$ is close to unity and the pressure-dependent flow $\Phi_{v}$ goes to zero, so that the Knudsen flow $\Phi_{k}$ accurately describes the observed flow as we enter the free molecular flow regime. Conversely, as pressure increases, the viscous flow $\Phi_{v}$ increases and the added flow becomes negligible.

By applying these equations to acetylene gas diffusing inside a microstructured fiber, the diffusion coefficient can be calculated for a given hole diameter and average pressure $\bar{P}$. This is done in Fig. 2 , which shows that the diffusion rate can be significantly improved by increasing the hole diameter.

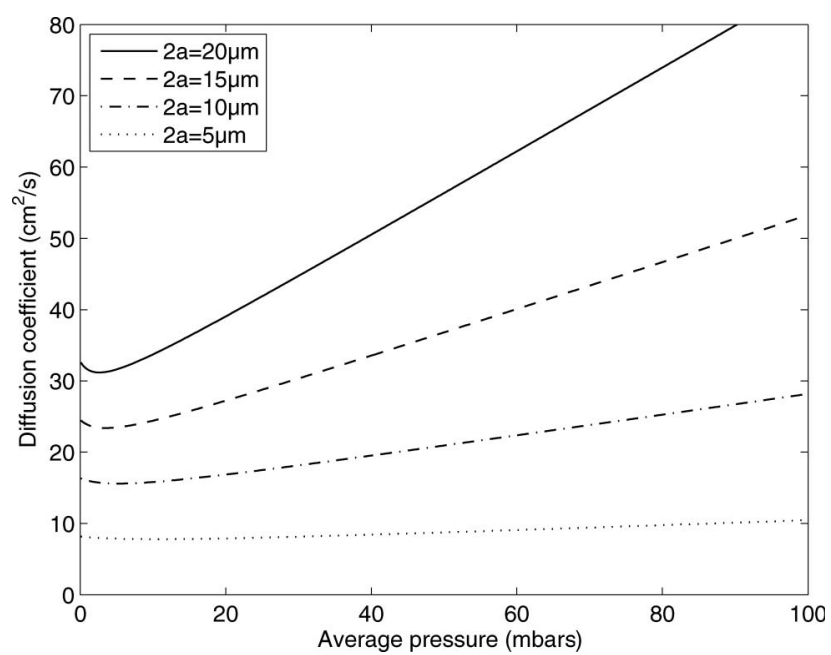

Fig. 2. Calculated diffusion coefficient of acetylene gas for several hole diameters in the slip-flow regime according to Eqs. (11) and (12).

\section{Gas-Filling Dynamics}

The distribution of the molecule density $n$ along the fiber length during the gas-filling process is obtained by solving the two partial differential equations presented in Section 2 (Eqs. (1) and (2)). By integrating the corresponding normalized pressure distribution over the fiber length $L$, one can obtain [15]

$$
\frac{P(t)}{P_{0}}=1-\frac{8}{\pi^{2}} \sum_{j=1,3,5}^{\infty} \frac{1}{j^{2}} \exp \left[-\left(\frac{j \pi}{\xi L}\right)^{2} D t\right],
$$

where $\xi$ is a geometrical factor depending on the filling conditions. It is equal to 2 for gas diffusing inside a fiber from one end and equal to unity for gas diffusing from both ends of the fiber. Moreover, Eq. (13) is valid only if the diffusion coefficient is constant during the filling process, which is the case in the free molecular flow regime or for the special case of trace-gas diffusion inside air-filled cladding holes. Because the diffusion process is determined by the slip-flow regime for pure gas diffusing in vacuumed microstructured fibers, the diffusion coefficient is no longer constant. Instead of using numerical solutions to accurately describe the filling process [12], we will approximate the diffusion coefficient as being constant throughout the filling process and the parameter $D$ in Eq. (13) will represent the averaged filling rate in any type of fiber for a given average pressure $\bar{P}$.

The filling time is here arbitrarily defined as the time required for the pressure $P$ in the fiber to reach $85 \%$ of the equilibrium pressure $P_{0}$ :

$$
P=85 \% P_{0} .
$$

This definition is justified by considering that typical fiber lengths can reach up to $20 \mathrm{~m}$ and that waiting for complete gas filling would actually take an infinite time. The choice of $85 \%$ is therefore a compromise between time consumption and reasonable 
filling along the fiber. With the help of Eq. (13), our analytical model for the filling time $t_{\text {fill }}$ of gaseous species inside PCFs is

$$
t_{\text {fill }}=\frac{(\xi L)^{2}}{\pi^{2} D} \ln \left[\frac{\pi^{2}}{8} \times \frac{P_{0}}{P_{0}-P}\right]
$$

and can be applied to any fiber geometry or length. The diffusion coefficient is calculated using

$$
D=\frac{a^{2} \bar{P}}{8 \eta}+Z\left(K_{n}\right) \frac{2}{3} a \bar{v},
$$

with the help of Eqs. (3), (5), ()), and (12) and the molecular mass equal to $2 \overline{6} .04 \mathrm{~g} / \mathrm{mol}$ for acetylene gas [16]. The average pressure is here defined as $\bar{P}=$ $\frac{2}{3} P_{0}$ to take into account the exponential profile of the filling process as it increases the average pressure to more than just $50 \%$ of the equilibrium pressure.

The comparison with experimental filling times is then performed by monitoring the gas-filling process in various types of microstructured fibers. From an experimental point of view, the average pressure inside a fiber during gas filling can be retrieved by monitoring the decreasing intensity $I(t, \nu)$ of an absorption line as a function of time:

$$
\frac{P(t)}{P_{0}}=\frac{\int \ln \left(I(t, \nu) / I_{0}(\nu)\right) d \nu}{\int \ln \left(I_{\text {fill }}\left(t_{\text {fill }}, \nu\right) / I_{0}(\nu)\right) d \nu} .
$$

This is done in the following experimental sections, where experimental and predicted filling times of several fiber samples are compared.

\section{Experimental Procedure}

The fiber samples were first spliced at one end to standard ITU-T G.652 single-mode fibers (SMFs) using an arc fusion splicer. A dispersion-compensating fiber (DCF) with an $\sim 4 \mu \mathrm{m}$ core diameter was spliced between the SMFs and the highly nonlinear PCFs to progressively match the mode-field diameters. The typical splice loss between the SMFs and DCFs is $1 \mathrm{~dB}$, compared to $2-3 \mathrm{~dB}$ for splices between DCFs and PCFs. These concatenated fibers were then placed inside a sealed gas chamber and vacuum was maintained for 4-8 days with the help of a turbo vacuum pump to let air fully evacuate the microstructured holes.

Acetylene gas was then introduced inside the chamber and the filling process was monitored using absorption lines in the $\nu_{1}+\nu_{3}$ rotational-vibrational band of ${ }^{12} \mathrm{C}_{2} \mathrm{H}_{2}$ centered at $\lambda=1.526 \mathrm{~nm}$. The injection current of a distributed-feedback laser diode

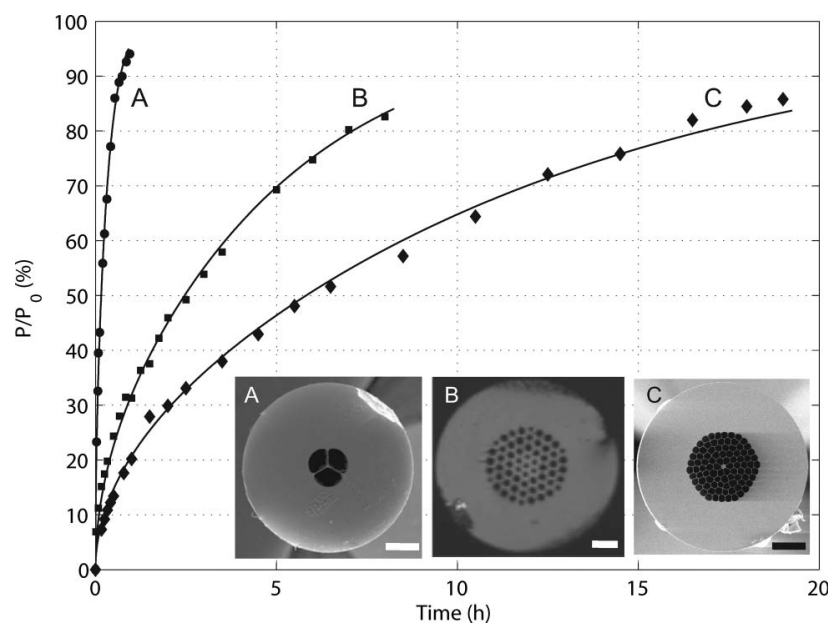

Fig. 3. Measured average pressure as a function of time in A, a suspended-core PCF; B, a microstructured-core PCF; and C, a solid-core PCF. The black curves represent nonlinear fittings of Eq. (13) from which experimental diffusion coefficients can be obtained. All scale bars represent $20 \mu \mathrm{m}$.

was continuously modulated to sweep the laser line across the absorption line. The wavelength information was retrieved with a Burleigh WA-1000 wavemeter, and a pressure gauge monitored the vacuum conditions inside the gas chamber.

\section{Results and Analysis}

This section presents the results of the gas-filling experiments in the various fiber samples. The samples were chosen to cover various fiber geometries: an airsuspended silica core surrounded by three large cladding holes, a microstructured germanium-doped core with three added layers of cladding holes, and a pure silica core surrounded by five layers of holes (insets A, B, and C, respectively, in Fig. 3). For each fiber sample, the hole diameter is defined as the smallest diameter that can be inscribed inside a hole over which the optical field is significantly present. Figure 3 shows the filling process of acetylene gas inside the three fiber samples for a filling pressure of 77 mbars with the corresponding experimental filling times presented in Table 1 .

As seen in Table 1, the temporal response of a fiber gas sensor can be predicted for any fiber geometry with at least $85 \%$ accuracy by using the simplified Eq. (15). Moreover, the relation between diffusion coefficient and pressure [i.e., Eq. (11)] was verified by filling a given fiber sample (here a suspended-core fiber) at various filling pressures and then retrieving experimental diffusion coefficients. As illustrated in Fig. 4, the experimentally determined values

\begin{tabular}{|c|c|c|c|c|c|c|}
\hline Fiber & Geometry & Length & Hole Diameter & Predicted Filling Time & Experimental Filling Time & Accuracy \\
\hline A & Suspended core & $2.7 \mathrm{~m}$ & $11.8 \mu \mathrm{m}$ & $35 \mathrm{~min}$ & $31 \min \pm 5 \min$ & $88 \%$ \\
\hline B & Microstructured core & $5.4 \mathrm{~m}$ & $3.4 \mu \mathrm{m}$ & $10 \mathrm{~h} 06 \mathrm{~min}$ & $9 \mathrm{~h} \pm 1 \mathrm{~h}$ & $90 \%$ \\
\hline $\mathrm{C}$ & Solid core & $9.2 \mathrm{~m}$ & $5.4 \mu \mathrm{m}$ & $18 \mathrm{~h} 12 \mathrm{~min}$ & $18 \mathrm{~h} 36 \min \pm 30 \mathrm{~min}$ & $97 \%$ \\
\hline
\end{tabular}
show reasonably satisfactory agreement with the

Table 1. Characteristics of the Microstructured Fibers 


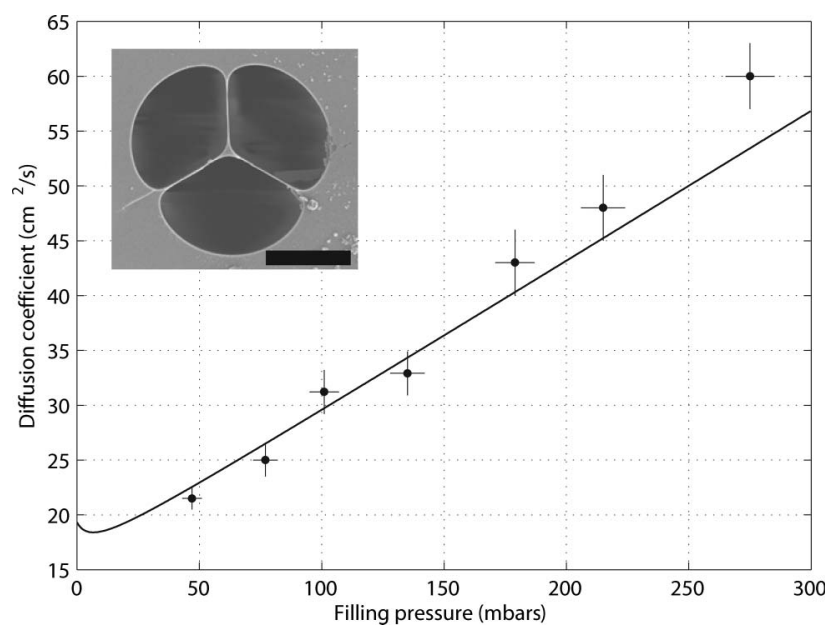

Fig. 4. Calculated diffusion coefficient for acetylene gas diffusing in suspended-core fibers ( $11.8 \mu \mathrm{m}$ hole diameter; scale bar, $10 \mu \mathrm{m})$ with average pressures taken as $2 / 3$ of the filling pressures. The data points represent experimental diffusion coefficients obtained using Eq. (11).

calculated ones. However, for filling pressures higher than 200 mbars, larger discrepancies can be seen. This is due to our analytical model, as the approximation for a constant diffusion coefficient is less accurate for higher pressures. Finally, the uncertainty in the measured $D$ values is due to normalization difficulties related to the oscillating background pattern often seen in PCF spectra [17], combined with the difficulties in evaluating the equilibrium pressure $P_{0}$.

\section{Ideal Gas-Filling Conditions}

In addition to being used as chemical sensors to detect hazardous gases, microstructured fibers can be filled with low-pressure gases to make all-fiber gas cells $[18,19]$. As the linewidth of absorption lines depends on gas pressure, the fibers usually contain pure gas at pressures of less than 200 mbars to maintain high wavelength accuracy. However, when gas chambers or similar devices are used for fiber fillings, the gas pressure inside the chamber will increase with time due to three main factors: virtual or real leaks, outgassing of molecules at low pressure, or back-streaming from the vacuum pump [20]. As a result, the gas leakage rate together with long filling times will introduce impurities into the gas cell. Figure 5 presents the predicted filling time of acetylene gas in microstructured fibers for a typical filling pressure of 100 mbars for any combination of hole diameter and fiber length.

Limiting the filling time according to a few selected criteria then secures the efficiency of the gas cell. Because the addition of impurities in the gas cell increases the width of the absorption line $\Delta \nu_{V}$ via the Lorentzian absorption linewidth $\Delta \nu_{L}$ according to the empirical relation

$$
\Delta \nu_{V}=0.5346 \Delta \nu_{L}+\sqrt{0.2169 \Delta \nu_{L}^{2}+\Delta \nu_{G}^{2}},
$$

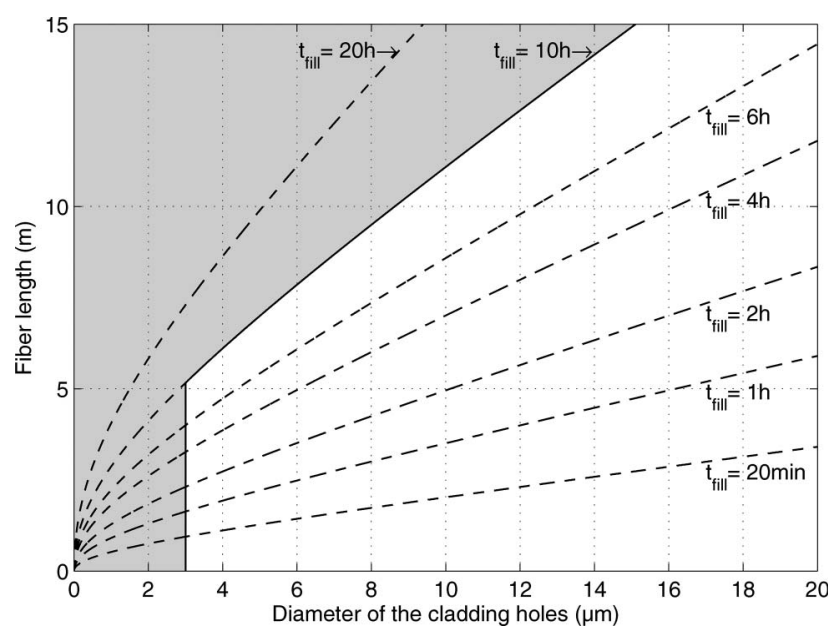

Fig. 5. Contours of constant filling time are plotted in the $2 a-L$ plane for a filling pressure of 100 mbars according to Eqs. (11) and (15) $\left(P / P_{0}=85 \%\right)$.

where $\Delta \nu_{G}$ is the Gaussian linewidth, allowing a maximum 5\% linewidth broadening due to impurities will yield a maximum impurity percentage of $\sim 10 \%$ for contamination by air (see [21] for broadening coefficients of acetylene in various buffer gases and [22] for numerical values of the Gaussian linewidths). According to a measured gas leakage rate of $1 \mathrm{mbar} / \mathrm{h}$, this corresponds to a maximum allowed filling time of $10 \mathrm{~h}$ for a 100 mbars filling pressure. In addition, by considering minimum fiber lengths of $5 \mathrm{~m}$ to ensure a minimal absorption efficiency according to the Beer-Lambert absorption law, a microstructured fiber should have a minimum hole diameter of $3 \mu \mathrm{m}$. These restrictions for the fiber characteristics are illustrated as shaded regions in Fig. 5. This is another indication that the gas flow dynamics is a crucial matter to consider when designing microstructured fibers for gas detection and all-fiber gas cell applications.

\section{Conclusion}

Helpful expressions predicting gas-filling times in PCFs have been proposed in this paper. Based on the theory of gas diffusion, our model can be readily applied to any fiber geometry or length, as confirmed by the experimental results. By studying the gasfilling process for various pressures inside a given fiber sample, we could experimentally confirm the pressure dependence of the diffusion coefficient and, therefore, the relation between filling pressure and predicted filling time as expected in the slip-flow regime. Conversely, by monitoring the gas-filling process in fibers, parameters such as the hole diameter and the gas viscosity can be estimated. This study of the gas flow dynamics can be used as a metrological tool to characterize PCFs and gas species.

The authors thank University Marie-Curie Skłodowskiej (UMCS) in Lublin, Poland, for providing the suspended-core fibers in the framework of COST 299 Action "FIDES". They also thank Xia Yu from 
Nanyang Technological University (NTU), Singapore and Kyunghwan Oh from Yonsei University in Seoul, South Korea, for providing the microstructured fibers. This work is funded by the European Space Agency (ESA) through grant 20200/06/NL/PA and by the Swiss National Office for Education and Research through project COST C06.0015.

\section{References}

1. J. M. Fini, "Microstructure fibres for optical sensing in gases and liquids," Meas. Sci. Technol. 15, 1120-1128 (2004).

2. C. M. B. Cordeiro, M. A. R. Franco, G. Chesini, E. C. S. Barretto, R. Lwin, C. H. B. Cruz, and M. C. J. Large, "Microstructured-core optical fibre for evanescent sensing applications," Opt. Express 14, 13056-13066 (2006).

3. P. S. Light, F. Couny, Y. Y. Wang, N. V. Wheeler, P. J. Roberts, and F. Benabid, "Double photonic bandgap hollow-core photonic crystal fiber," Opt. Express 17, 16238-16243 (2009).

4. L. Dong, B. K. Thomas, and L. Fu, "Highly nonlinear silica suspended core fibers," Opt. Express 16, 16423-16430 (2008).

5. S.-G. Li, S.-Y. Liu, Z.-Y. Song, Y. Han, T.-L. Cheng, G.-Y. Zhou, and L.-T. Hou, "Study of the sensitivity of gas sensing by use of index-guiding photonic crystal fibers," Appl. Opt. 46, 5183-5188 (2007).

6. A. S. Webb, F. Poletti, D. J. Richardson, and J. K. Sahu, "Suspended-core holey fiber for evanescent-field sensing," Opt. Eng. 46, 010503 (2007).

7. T. G. Euser, J. S. Y. Chen, M. Scharrer, P. St. J. Russell, N. J. Farrer, and P. J. Sadler, "Quantitative broadband chemical sensing in air-suspended solid-core fibers," J. Appl. Phys. 103, 103108 (2008).

8. F. Couny and F. Benabid, "Optical frequency comb generation in gas-filled hollow core photonic crystal fibres," J. Opt. A Pure Appl. Opt. 11, 103002 (2009).

9. Y. L. Hoo, W. Jin, C. Shi, H. L. Ho, D. N. Wang, and S. C. Ruan, "Design and modeling of a photonic crystal fiber gas sensor," Appl. Opt. 42, 3509-3515 (2003).
10. T. Ritari, J. Tuominen, H. Ludvigsen, J. Petersen, T. Sørensen, T. Hansen, and H. Simonsen, "Gas sensing using air-guiding photonic bandgap fibers," Opt. Express 12, 4080-4087 (2004).

11. N. Gayraud, Ł. W. Kornaszewski, J. M. Stone, J. C. Knight, D. T. Reid, D. P. Hand, and W. N. MacPherson, "Mid-infrared gas sensing using a photonic bandgap fiber," Appl. Opt. 47, 1269-1277 (2008).

12. J. Henningsen and J. Hald, "Dynamics of gas flow in hollow core photonic bandgap fibers," Appl. Opt. 47, 2790-2797 (2008).

13. S. Dushman and J. M. Lafferty, Scientific Foundations of Vacuum Technique (Wiley, 1962).

14. R. Cunningham and R. Williams, Diffusion in Gases and Porous Media (Plenum, 1980).

15. W. Jost, Diffusion in Solids, Liquids, Gases (Academic, 1970).

16. C. L. Yaws, Handbook of Transport Property Data: Viscosity, Thermal Conductivity, and Diffusion Coefficients of Liquids and Gases (Gulf, 1995).

17. J. Henningsen, J. Hald, and J. C. Peterson, "Saturated absorption in acetylene and hydrogen cyanide in hollow-core photonic bandgap fibers," Opt. Express 13, 10475-10482 (2005).

18. S. Chin, I. Dicaire, J.-C. Beugnot, S. Foaleng-Mafang, M. Gonzalez-Herraez, and L. Thévenaz, "Material slow light does not enhance Beer-Lambert absorption," in Slow and Fast Light (Optical Society of America, 2009), paper SMA3.

19. P. S. Light, F. Couny, and F. Benabid, "Low optical insertionloss and vacuum-pressure all-fiber acetylene cell based on hollow-core photonic crystal fiber," Opt. Lett. 31, 2538-2540 (2006).

20. J. O'Hanlon, A User's Guide to Vacuum Technology (Wiley, 2003).

21. K. S. Bond, N. D. Collett, E. P. Fuller, J. L. Hardwick, E. E. Hinds, T. W. Keiber, I. S. G. Kelly-Morgan, C. M. Matthys, M. J. Pilkenton, K. W. Sinclair, and A. A. Taylor, "Temperature dependence of pressure broadening and shifts of acetylene at $1550 \mathrm{~nm}$ by He, Ne, and Ar," Appl. Phys. B 90, 255-262 (2008).

22. W. C. Swann and S. L. Gilbert, "Pressure-induced shift and broadening of 1510-1540 nm acetylene wavelength calibration lines,” J. Opt. Soc. Am. B 17, 1263-1270 (2000). 